\section{PWE-038 OUTCOMES OF AN ANAEMIA SERVICE EVALUATION USING THE IBD REGISTRY}

${ }^{1}$ William Waddingham, ${ }^{2}$ Lisa McCluskey ${ }^{*},{ }^{3}$ Benjamin Crooks, ${ }^{3}$ Vanessa Davies, ${ }^{4}$ Suranga Dharmasiri, ${ }^{4}$ Angel Castro Silva, ${ }^{2}$ Kjersti Haukenes, ${ }^{5}$ Josh Abbott, ${ }^{6}$ David Oliver, ${ }^{3}$ Paul Knight, ${ }^{7}$ Matthew Johnson, ${ }^{1}$ Stuart Bloom, ${ }^{4}$ Fraser Cummings. ${ }^{1}$ University College London Hospitals, London, UK; ${ }^{2}$ Pharmacosmos UK Ltd, Reading, UK; ${ }^{3}$ Wythenshawe Hospital (UHSM), Manchester, UK; ${ }^{4}$ University Hospital Southampton, Southampton, UK; ${ }^{5}$ Dovetail, London, UK; ${ }^{6}$ James Cook University Hospital, Middlesborough, UK; ${ }^{7}$ Luton and Dunstable University Hospital, Luton, UK

\subsection{6/gutjnl-2018-BSGAbstracts. 170}

Introduction Iron deficiency (ID) and iron deficiency anaemia (IDA) are frequent complications of Crohn's disease (CD) and Ulcerative Colitis (UC). Assessing iron status in IBD patients can be challenging as tests may be unreliable in the presence of inflammation. European Crohn's and Colitis Organisation (ECCO) guidelines state FBC, CRP and ferritin are the minimum to detect IDA.

The UK IBD audit of inpatient care found $56 \%$ of those with IDA did not receive iron, but the standard of care for IBD outpatients is unknown. IBD patients with IDA rarely have an iron deficit of $<1000 \mathrm{mg}$ iron and effective treatment for ID/IDA requires correct and sufficient dosing of iron. This project compares current practice with the ECCO guidelines.

Initial pilot data on ID/IDA diagnosis is presented from a Joint Working project using an adapted Webtool with anaemia specific parameters to determine the standard of care for IBD outpatients.

Methods 20 consecutive consented patients (10 CD, 10 UC) were recruited at 5 sites and followed for around 12 months.

Anaemia: $\mathrm{Hb}<120 \mathrm{~g} / \mathrm{L}$ or $<130 \mathrm{~g} / \mathrm{L} \sigma^{\wedge}$

ID: $\mathrm{MCV}<80 \mathrm{fl}$ AND/OR ferritin $<30 \mathrm{ug} / \mathrm{L}$ if CRP $\leq 5 \mathrm{mg} / \mathrm{L}$ OR ferritin $\geq 30 \mathrm{ug} / \mathrm{L}$ to $<100 \mathrm{ug} / \mathrm{L}$ if CRP $>5 \mathrm{mg} / \mathrm{ml}$ AND/OR TSAT $<20 \%$

IDA: Anaemia and ID

Results Baseline data were available for 94 patients: 45 (48\%) male and 49 (52\%) female, 47 (50\%) with CD and 45 (48\%) with UC ( 2 patients with unidentified IBD) mean age 46.5 years.

82 patients had $\geq 1$ recorded haematinic and 18 of these (22\%) had 26 anaemic episodes, with 10 (56\%, 5 UC and $5 \mathrm{CD})$ and $14(54 \%)$ of these patients and episodes being IDA. 23 (28\%, $8 \mathrm{CD}, 14 \mathrm{UC}, 1$ undefined IBD) patients experienced 34 episodes of ID.

$136 \mathrm{Hb}$ results were recorded, but only 78 (57\%) were combined with ferritin plus CRP.

Conclusions Most cases of anaemia were IDA, and more episodes of ID than IDA were found. An equal number of CD and UC patients had IDA, but non-anaemic ID was more common in UC than CD patients. As only $57 \%$ of haematinic tests fulfilled the minimum requirement to detect ID in anaemic IBD patients ( $\mathrm{Hb}$ combined with ferritin plus CRP) ID/ IDA may be significantly underdiagnosed conditions in IBD. However, these findings are limited due to the small, realworld, dataset.

An adapted Registry Webtool may allow easy data collection though there are challenges in completing data input during consultations. Iron status could therefore be better monitored if haematinics were a default part of the IBD Registry dataset, allowing for quality improvement.
PWE-039 FMT AS A TREATMENT FOR IBD: A NATIONAL SURVEY OF GASTROENTEROLOGISTS IN THE UK

${ }^{1}$ James Mcilroy*, ${ }^{1}$ Niharika Nalagatla, ${ }^{2}$ Richard Hansen, ${ }^{3}$ Ailsa Hart, ${ }^{1}$ Georgina Hold. ${ }^{1}$ University Of Aberdeen, Aberdeen, UK; ${ }^{2}$ Royal Hospital for Children, Glasgow, UK; ${ }^{3}$ St Mark's Hospital, London, UK

\subsection{6/gutjnl-2018-BSGAbstracts. 171}

Introduction Interest in the use of faecal microbiota transplantation (FMT) as a therapeutic treatment in inflammatory bowel disease (IBD) has increased following promising outcomes in patients with Clostridioides difficile infection (CDI). To date, 4 double blind randomised control trials have been conducted investigating the efficacy of FMT in patients with ulcerative colitis (UC). Whilst research exploring clinician awareness and attitude towards the use of FMT in CDI has been carried out, data for IBD is currently lacking, despite growing patient and clinician interest in the treatment. The primary aim of this survey was to assess the perceptions of gastroenterologists and current practice relating to FMT as a treatment for IBD in the UK.

Methods The survey was developed using Snap Survey software, following literature review and author consensus. It was distributed amongst UK-based gastroenterology trainees and consultants through the British Society of Gastroenterology and British Society of Paediatric Gastroenterology, Hepatology and Nutrition e-newsletters, and at the BSG Conference in June 2017.

Results In total, 61 respondents completed the survey including pre-subspecialty trainees, gastroenterology specialists, associate specialists and consultants. Almost all $(95 \% ; n=58)$ respondents stated that they had heard of FMT being used as a treatment for IBD prior to participating in the survey. Based on current evidence, 34\% $(n=21)$ of respondents would consider using FMT in patients with IBD, 26\% $(n=16)$ would not and 39\% $(n=24)$ were undecided. A total of $43 \%(n=26)$ respondents said that a patient had expressed interest in FMT and a small proportion $(10 \% ; n=6)$ said that they were aware of a patient that has undertaken FMT on their own without medical supervision. When asked to rank routes of delivery in terms of preference, nasogastric tube was the least preferred route $(39 \% ; \mathrm{n}=24)$ and oral capsule was the most preferred route $(34 \% ; \mathrm{n}=21)$.

Conclusions A clear majority of UK gastroenterologists recognise FMT as a potential treatment for IBD, however uptake is limited. A significant proportion of clinicians would consider FMT in IBD based on currently available evidence and the majority would consider entering patients into clinical trials. Orally-delivered encapsulated FMT is the preferred route of administration and future work should explore the utility and efficacy of this route.

\section{PWE-040 MICROSCOPIC COLITIS AND PROTON PUMP INHIBITORS - USE OF THE NULL HYPOTHESIS}

Alex Moran*. North Devon District Hospital, Barnstaple, UK

10.1136/gutjnl-2018-BSGAbstracts. 172

Introduction Many studies have shown a strong association with proton pump inhibitor (PPI) usage and the development 
of microscopic colitis (MC). Due to the association of PPIs with diarrhoea there is a risk of confounding bias due to increased investigation with colonoscopy and biopsies. This has resulted in a controversy in case-control studies with regard to using a control group from the background population (BP) or a control group with investigated chronic diarrhoea (ICD). This abstract evaluates the use of the null hypothesis for MC and PPIs with relation to published casecontrol studies of MC and PPIs and with discussion of potential mechanisms

Methods The Null Hypothesis for MC and PPIs can be categorised according to MC being largely clinical and investigated or a largely subclinical uninvestigated disease.

Hypothesis 1: PPIs and MC are unrelated and MC is always overt and investigated by colonic biopsies.

Hypothesis 2: PPIs and MC are unrelated and MC is always subclinical.

For age and sex matched groups - those with clinical MC (hypothesis 1) that are detected will have the same percentage on PPIs as those from the background population (BP), whereas those with subclinical MC (hypothesis 2) that are detected will have the same percentage on PPIs as those with investigated chronic diarrhoea (ICD)

Results There are 6 published case-control studies and a recent abstract that provide adjusted odds ratios (AORs)/odds ratios (ORs) for PPIs and MC. Some of the larger studies have divided MC patients into the two subgroups; collagenous colitis (CC) and lymphocytic colitis (LC).

\begin{tabular}{|c|c|c|c|c|c|c|c|}
\hline & & $\mathrm{n}$ & $\%$ & $\mathrm{BP} \%$ & $\mathrm{AOR} /$ & IDC\% & $\mathrm{AOR}$ \\
\hline & & & PPIs & PPIs & $\mathrm{OR}$ & PPIS & OR \\
\hline \multirow{3}{*}{$\begin{array}{l}\text { Bonderup } \\
2014\end{array}$} & $\mathrm{CC}$ & 3474 & 55.7 & 14.2 & 7.04 & & 3.47 \\
\hline & & & & & & & \\
\hline & LC & 2277 & 36.7 & 12.9 & 3.37 & & 3.57 \\
\hline Bonderup & $\mathrm{CC}$ & 6254 & & & 8.75 & & \\
\hline \multicolumn{8}{|l|}{2017} \\
\hline (abstract) & LC & 4398 & & & 5.03 & & \\
\hline \multirow[t]{2}{*}{ Banares 2013} & $\mathrm{CC}$ & 120 & 33.3 & 14.8 & & & \\
\hline & LC & 70 & 31.4 & 14.8 & & & \\
\hline Kesthelyi 2010 & $\mathrm{MC}$ & 95 & 37.9 & 12.6 & 4.5 & & \\
\hline Pascua 2010 & $\mathrm{MC}$ & 26 & 12 & 45 & 0.21 & 34 & 0.29 \\
\hline Verhaugh & MC & 1211 & 41.8 & 17.5 & 3.79 & & \\
\hline \multicolumn{8}{|l|}{2016} \\
\hline Masclee 2015 & $\mathrm{MC}$ & 218 & 30 & 2.4 & 7.3 & 6.3 & 10.6 \\
\hline
\end{tabular}

Conclusions MC is unlikely to be always investigated and there is some evidence that MC can be detected in asymptomatic individuals. However, it is also unlikely that $\mathrm{MC}$ is largely subclinical and not investigated. All but one case-control studies have shown increased AORs for MC and PPIs for BP controls and similarly in 2 of 3 studies for ICD controls. The only study not showing an association included only 26 cases with MC and a very high usage of PPIs in the control BP of $45 \%$. The mechanisms of how PPIs may cause MC are unclear but theories include increased intestinal epithelial permeability, alteration of colonic bacterial flora and increased production of collagen by colonocytes. The association of MC with medications including PPIs should not be ignored and cessation of potentially causative medications requires consideration.

\section{PWE-041 ALTERATION IN SMALL BOWEL MOTILITY, GUT PEPTIDES AND PATIENT'S SYMPTOMS IN ACTIVE CROHN'S DISEASE}

${ }^{1,2}$ Gordon Moran*, ${ }^{1,2}$ Asseel Khalaf, ${ }^{2,3}$ Caroline Hoad, ${ }^{4}$ Alex Menys, ${ }^{2}$ Shellie Radford, ${ }^{2}$ Adam Nowak, ${ }^{1,2}$ Stephen Paparo, ${ }^{2}$ Melanie Lingaya, ${ }^{2}$ Yirga Falcone, ${ }^{2}$ Gulzar Singh, ${ }^{4}$ Stuart Taylor, ${ }^{1,2}$ Robin Spiller, ${ }^{3}$ Penny Gowland, 1,2 Luca Marciani. 'Nottingham Digestive Diseases Centre, School of Medicine, University of Nottingham, Nottingham, UK; ${ }^{2}$ NIHR Biomedical Research Centre in Gastrointestinal and Liver Diseases at Nottingham University Hospitals NHS Trust and the University of Nottingham, Nottingham, UK; ${ }^{3}$ Sir Peter Mansfield Imaging Centre, School of Physics and Astronomy, University of Nottingham, Nottingham, UK; ${ }^{4}$ Centre for Medical Imaging, Division of Medicine, UCL, London, UK

\subsection{6/gutjnl-2018-BSGAbstracts. 173}

Introduction Intestinal inflammation in Crohn's disease (CD) is associated with an increase in Polypeptide YY (PYY), Glucagon-like peptide 1 (GLP-1) and cholecystokinin (CCK). CD patients experience postprandial fullness and nausea. These symptoms may be linked to the increase in plasma gut peptides levels and alterations in intestinal motility.

Our aims are to quantify gut peptide, small bowel motility and patient symptom response to a standard test meal using Magnetic Resonance Imaging (MRI).

Methods Subjects underwent baseline and postprandial MRI scans, symptom questionnaires and blood sampling (GLP-1, PYY, CCK) at intervals for $270 \mathrm{~min}$ following a test meal: soup (400 g) (Heinz, Wigan, UK); (kcal) 51, protein $1.5 \mathrm{~g}$, carbohydrate $4.7 \mathrm{~g}$, fat $2.9 \mathrm{~g}$ per $100 \mathrm{~g}$.

MRI scans were performed using a $1.5 \mathrm{~T}$ Philips Achieva MRI scanner. Gastric volume, small bowel water content (SBWC) and small bowel motility were assessed using MRI. Patients also underwent a standard contrast enhanced clinical MR enterography (MRE) and MaRIA score applied to quantify disease activity. All subjects gave informed written consent. Trial registration number: NCT03052465. Data is presented as mean \pm SEM.

Results CD patients showed a significantly $(\mathrm{p} \leq 0.05)$ slower

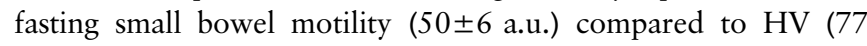
\pm 10 a.u.). Postprandial SBWC was significantly greater in CD than $\mathrm{HV}$ (measured as area under the curve CD: 18452, HV: 13760, p $\leq 0.05$ ). Fasting PYY (CD: $236 \pm 16$ pg/mL, HV: 118 $\pm 11 \mathrm{pg} / \mathrm{mL}, \mathrm{p} \leq 0.0001)$ and GLP-1 (CD: $50 \pm 8 \mu \mathrm{g} / \mathrm{mL}$ HV: 13 $\pm 3 \mu \mathrm{g} / \mathrm{mL}, \mathrm{p} \leq 0.0001)$ were significantly higher in CD compared to $\mathrm{HV}$ with this difference persisting at each time point of the study $(p \leq 0.0001)$. The meal induced a significant increase $(p \leq 0.0001)$ in fullness, bloating and abdominal pain scores in patients $(28 \pm 4 \mathrm{~mm}, 22 \pm 3 \mathrm{~mm}$ and $12 \pm 2 \mathrm{~mm}$ respectively) compared to $\mathrm{HV}(12 \pm 4 \mathrm{~mm}, 3 \pm 3 \mathrm{~mm}$ and 1 $\pm 2 \mathrm{~mm}$ respectively). No differences were noted in gastric volumes, CCK concentration and postprandial motility.

Conclusion The decrease in fasting small bowel motility noted in $C D$ may be ascribed to the increased fasting GI peptides. Understanding the physiological changes in disease groups will allow us to identify the key biomarkers for pharmacological modulation to improve patient symptoms. 